\title{
G- AND C-BANDING IN THE AUSTRALIAN GRASSHOPPER PHAULACRIDIUM VITTATUM
}

\author{
G. C. WEBB* and M. WESTERMAN† \\ * Department of Population Biology, R.S.B.S., Australian National University, P.O. Box 475, \\ Canberra A.C.T., 2600, Australia; † Department of Genetics, La Trobe University, \\ Bundoora, Victaria 3083, Australia
}

Received 21.xii.77

\begin{abstract}
SUMMARY
The G- and G-banding patterns of Phaulacridium vittatum (Orthoptera : Acrididae) chromosomes have been investigated. The autosomes and sex chromosomes are similar in showing light staining procentric G-bands and dark staining procentric C-bands. One autosomal pair has a variable distal band. The C-banding pattern is similar in mitotic and meiotic cells. The long teleocentric B-chromosome is somewhat different in its staining behaviour, it is only slightly affected by G-banding but on G-banding it appears relatively more deeply staining than other members of the complement and shows some evidence of longitudinal differentiation into bands and interbands. The results provide no evidence for a scheme of evolutionary origin of the Bchromosome from the $\mathrm{X}$ or any other large chromosome. One egg pod was observed to contain two embryos having two different $\mathrm{B}$ 's - a long telocentric $\mathrm{B}^{\mathrm{T}}$ chromosome and a metacentric B-isochromosome. C-banding patterns suggest that the latter most probably arose from the former via misdivision of the centromere in one of the very early cleavage divisions of the embryo.
\end{abstract}

\section{INTRODUGTION}

To date few supernumerary (B) chromosomes have been investigated by means of chromosome banding techniques. Baverstock ct al. (1976) have shown that the B-chromosomes of a number of Australian native rodents stain deeply but uniformly over their whole length after C-banding. This pattern of C-banding is similar to that observed in most other mammals (see Baverstock, et al., 1977 for details). In contrast to the uniform C-banding pattern, G-banding of the B-chromosome of Uromys reveals a series of alternating bands along its length (Baverstock, personal communication).

In the Orthoptera Gallagher et al. (1973) showed that the banding pattern of each arm of the metacentric isochromosome B in Myrmeleotettix maculatus was similar to that of the telocentric X-chromosome from which it was postulated to be derived. This banding pattern was induced by a C-banding technique-no marked response being observed when trypsin G-banding was used (Hewitt, personal communication). A B-chromosome in the grasshopper Caledia captiva has been C-banded (Shaw and Wilkinson, unpublished) and showed a banding pattern similar to those seen on the autosomes described by Shaw et al. (1976). Somewhat spectacular banding patterns have been demonstrated for the B-chromosomes of the Australian plague locust Chortoicetes terminifera (see Webb, 1976; Webb and Komarowski, 1976). In this species the large B-chromosomes respond to both G- and C-banding techniques and a number of variant banding patterns have been found to exist in natural populations (Webb and Neuhaus, in preparation).

The present paper describes an attempt to obtain G- and C-banding 41/2-A 
patterns for the large B-chromosomes of Phaulacridium vittatum in the hope that these would clarify speculations on the possible evolutionary origin of these chromosomes.

\section{Materials and Methods}

Male and female $P h$. vittatum individuals were taken from the La Trobe University campus population towards the end of the summer season. It is known that at this time of the year the B-chromosome frequency in adults of this population is very high (Westerman and Dempsey, 1977). Mitotic metaphases were prepared from adults injected with colchicine or from embryos immersed in colchicine/saline. Air dried slides were made for trypsin G- and barium hydroxide C-banding using methods outlined in Webb (1976). The slides for C-banding treatments were left overnight on a hotplate at about $60^{\circ} \mathrm{C}$ prior to treatment. Banded meiotic chromosomes were also prepared from testes but these yielded little additional information.

\section{Results}

When mitotic interphase cells of Ph. vittatum are subjected to G- and Cbanding procedures the only features made visible are dark staining procentric masses often located together towards one side of the nucleus (fig. la). This pattern indicates a polarised orientation of centromeres seen in prophase (fig. lb). With the exception of a small lighter staining region adjacent to the centromere (fig. lc) the mitotic metaphase chromosomes of $P h$. vittatum show little differentiation along their length after G-banding treatment with trypsin, a finding which appears to be general for insect chromosomes except for the B-chromosome of Chortoicetes terminifera (Webb, 1976). Although one of the autosomes shows a distally located light staining region, none could be consistently distinguished on the basis of G-banding patterns nor could the sex chromosomes be identified other than on the basis of their length. The light staining regions observed after G-banding correspond with positively staining C-bands (fig. ld). Thus after C-banding procedures not only did the centromeric regions of both $\mathrm{X}$ and autosomes stain deeply, so too did one distal region which showed very variable staining intensity and is perhaps polymorphic for size in the population. The smallest variant of this distal band is so faintly stained as to appear absent in most preparations (compare figs. $1 \mathrm{~d}$ and $2 \mathrm{~d}$ ). Corresponding G-banding patterns were also obtained for meiotic chromosomes of $P$ h. vittatum (fig. le).

The $\mathrm{B}^{\mathrm{T}}$-chromosome of $P h$. vittatum is one of the largest chromosomes in the complement (Jackson and Cheung, 1967; Rowe and Westerman, 1974). Whilst it can be clearly seen in orcein stained male meiotic prophase cells as a uniformly dark staining mass whose coiling cycle closely follows that of the single allocyclic X-chromosome, neither $\mathrm{B}$ nor $\mathrm{X}$ can normally be distinguished in mitotic interphase cells. The B-chromosome cannot be identified in either G- or C-banded interphase cells (fig. la). At mitotic metaphase however the result is somewhat different. In contrast to the uniform staining pattern of the $\mathrm{X}$ and autosomes, following trypsin Gbanding the B-chromosome can be identified by the absence of a light staining region adjacent to the centromere and by a faint interstitial gap in the staining (see fig. lc). The dissimilarity of A- and B-chromosomes in $P h$. vittatum was even more marked after $\mathrm{C}$-banding procedures. Here the 
B-chromosome was not only more densely staining than either the $\mathrm{X}$ or autosomes at mitotic metaphase it also showed some evidence of differentiation into darker bands and interbands along its length (see fig. 1d). A similar differentiation is sometimes observed in extended B-chromosomes during metiotic prophase after lactopropionic orcein staining (fig. If).

Orcein stained meiotic preparations frequently show presence of a large secondary constriction in the proximal third of the X-chromosome of $P h$. vittatum (fig. 2a). Similar constrictions occur on the X-chromosome of all Phaulacridium species examined to date-Ph. nanum (fig. $2 \mathrm{~b}$ ), $P$ h. marginale (see fig. la, Westerman, 1975b) and probably in $P h$. sp3. The Bchromosomes of these four species also often shows a similar secondary constriction (see fig. 2c). This may be represented in the C-banded preparations of $P h$. vittatum by one of the darker cross bands on the B-chromosome.

TABLE 1

Karyotypes of the thirteen embryos observed in one egg pod from a female $\mathrm{Ph}$. vittatum

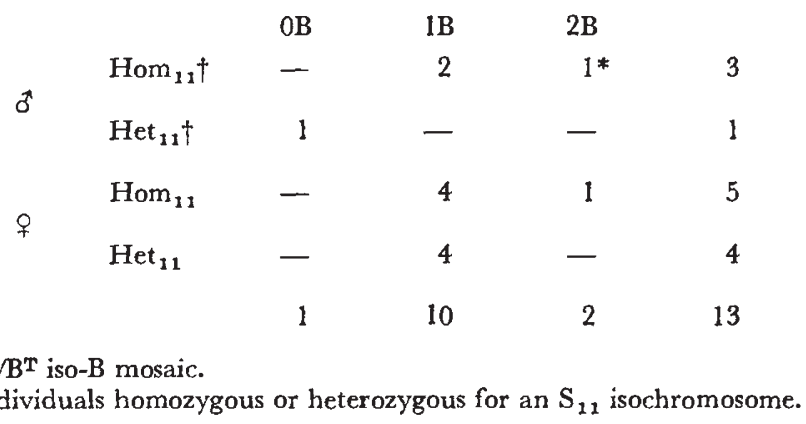

One egg pod obtained in the present study proved to be of great interest. The embryos from this pod were of three major types-those having no B-chromosomes, those having one B-chromosome and those having two B's (see table 1). Of these latter, one embryo was found to be a mosaic having some metaphases showing a single long telocentric $\mathrm{B}^{\mathrm{T}}$-chromosome, others having one long $\mathrm{B}^{\mathrm{T}}$ together with a long metacentric B-chromosome. The C-banding pattern showed that this chromosome was in fact an isochromosome (see fig. 2d). Such a situation could arise in an embryo containing two $\mathrm{B}^{\mathrm{T}}$-chromosomes as outlined in fig. 3. The absence of cells containing $2 \mathrm{~B}^{\mathrm{T}}$-chromosomes would suggest that the centric misdivision event giving rise to the $\mathrm{B}$-isochromosome must have occurred at a very early stage of development-possibly the first cleavage division. This long Bisochromosome is the first recorded instance of a misdivision involving a long B $^{\mathrm{T}}$-chromosome type in this genus and can be added to those Bchromosome types already known for Phaulacridium (see Westerman, 1977).

\section{Discussion}

With the exception of small dark staining proximal regions the Achromosomes of Ph. vittatum, like those of many insect species, showed little evidence of any longitudinal differentiation after either C-banding or trypsin G-banding. Indeed studies on the banding patterns of the related 

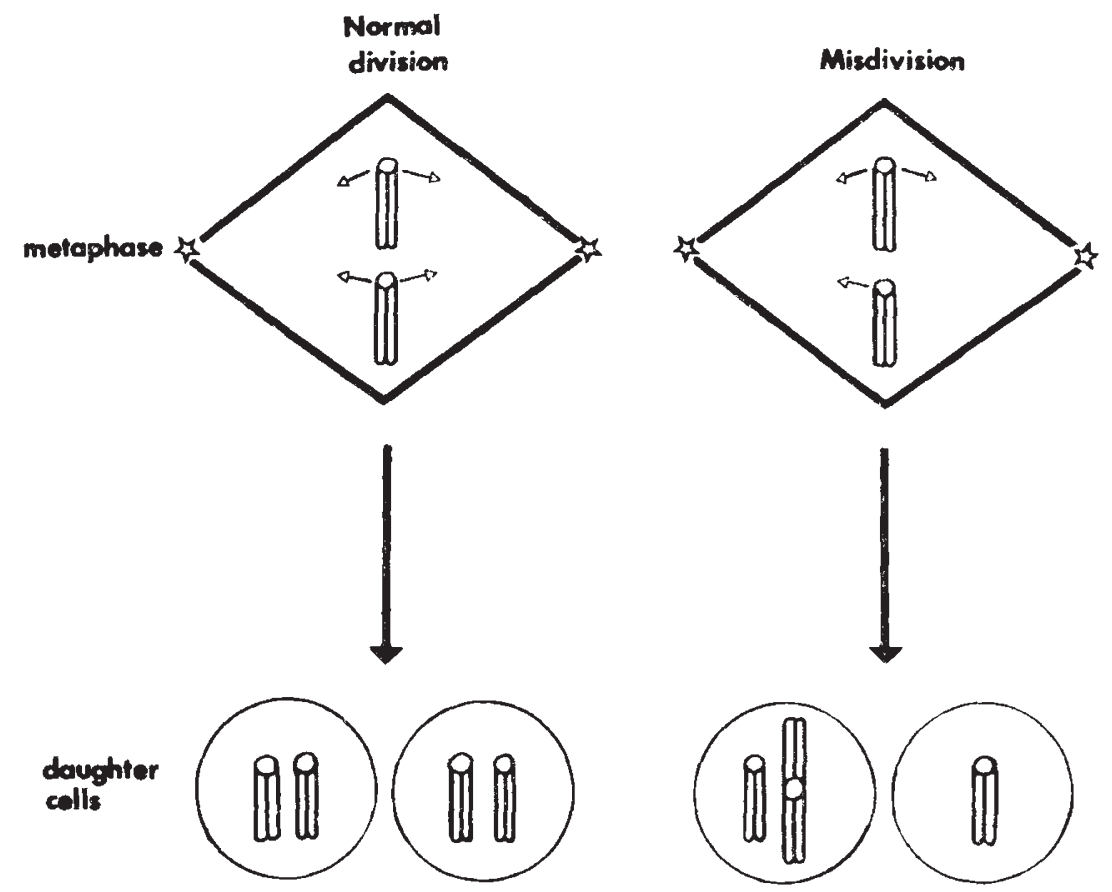

FIG. 3.-Possible mode of origin of the B-isochromosome in a Ph. vittatum embryo.

species $P h$. nanum, $P h$. marginale and $P h$. sp3. show a similar paucity of banding pattern for both mitotic and meiotic chromosomes (Westerman, in preparation). Although the occurrence of some paracentric inversions and/or changes in amounts of DNA per diploid nucleus cannot be ruled out, the great similarity of karyotypes seen in this genus suggests that there has been remarkably little change to the chromosomes accompanying speciation. The small size of the centric C-bands both in $P h$. vittatum and its related species also correlate with an observed absence of both satellite DNA's in neutral $\mathrm{C}_{3} \mathrm{Cl}$ gradients and of cryptic satellites following heavy metal/ $\mathrm{Cs}_{2} \mathrm{SO}_{4}$ centrifugation (Westerman, in preparation).

The B-chromosome of $P h$. vittatum does appear to behave differently from the A-chromosomes, particularly after C-banding when there is some evidence of darker bands along its length. It also differed from the $\mathrm{X}$ chromosome. Thus the present G- and C-banding patterns shed little light on the evolutionary origin of the B-chromosome. On a number of criteria, e.g. similarity of coiling patterns in male meiotic prophase, presence of similar secondary constrictions on $\mathrm{X}$ and B, etc. Westerman (1975a) has argued that $\mathrm{B}$-chromosomes in the genus Phaulacridium have probably arisen by non-disjunction from the X-chromosomes. It is of interest that in the present study no G- or G-bands were observed on the $\mathrm{X}$ other than those close to the centromere. This finding was somewhat unexpected in that the $\mathrm{X}$-chromosomes of all Phaulacridium species examined to date show large secondary constrictions in the proximal third of the chromosome when stained with orcein-as do their B-chromosomes. This dissimilarity of $\mathrm{C}$-banding patterns of $\mathrm{X}$ and $\mathrm{B}$-chromosomes in Ph. vittatum is in marked 

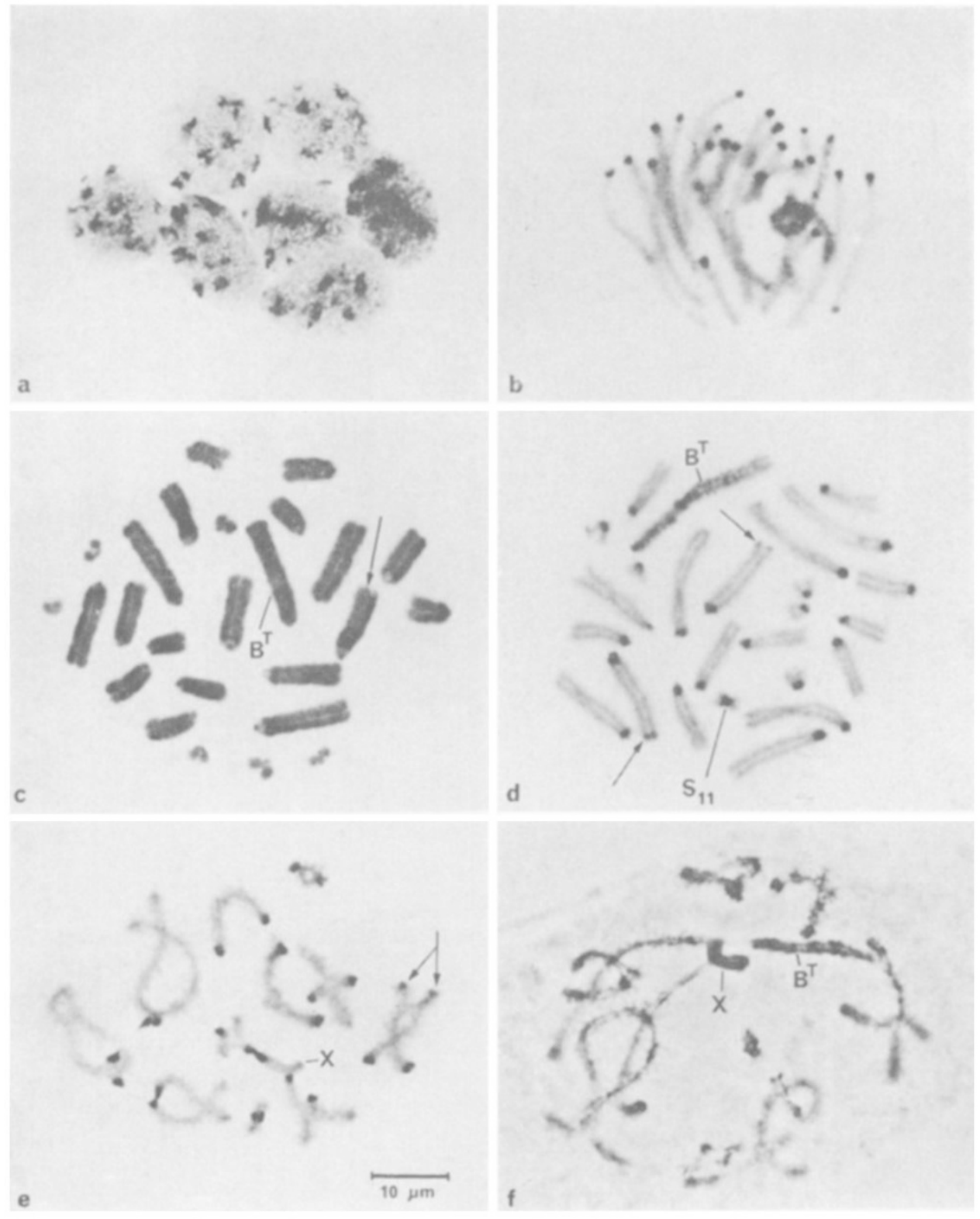

\section{Plate I}

Frg. 1.-C- and G-banded cells of Ph. vittatum, a-d and e have one $\mathrm{BT}^{\mathrm{T}}$-chromosome.

(a) C-banded interphase cells from an embryo showing distribution of pro-centric C-bands. The B is not obvious at interphase.

(b) C-banded embryonic prophase showing polarized orientation of the centromeres.

(c) G-banded mitotic metaphase from a female embryo. Note the procentric light staining region on most chromosomes and one distal light staining region (arrow). The B-chromosome has only a faint interstitial gap in its staining.

(d) C-banded metaphase from a female embryo. Note the dark staining procentric regions of all chromosomes. One pair has distal dark staining region (arrows). There is some differential banding of the $\mathrm{B}^{\mathrm{T}}$-chromosome. This individual is also heterozygous for an $\mathrm{S}_{11}$ isochromosome.

(e) C-banded diplotene in Ph. vittatum without a B chromosome. Note the procentric C-bands and the distal band on one bivalent (arrow).

(f) Orcein stained diplotene of $P h$. vittatum showing banded appearance of $\mathrm{B}^{\mathrm{T}}$ chromosome. 

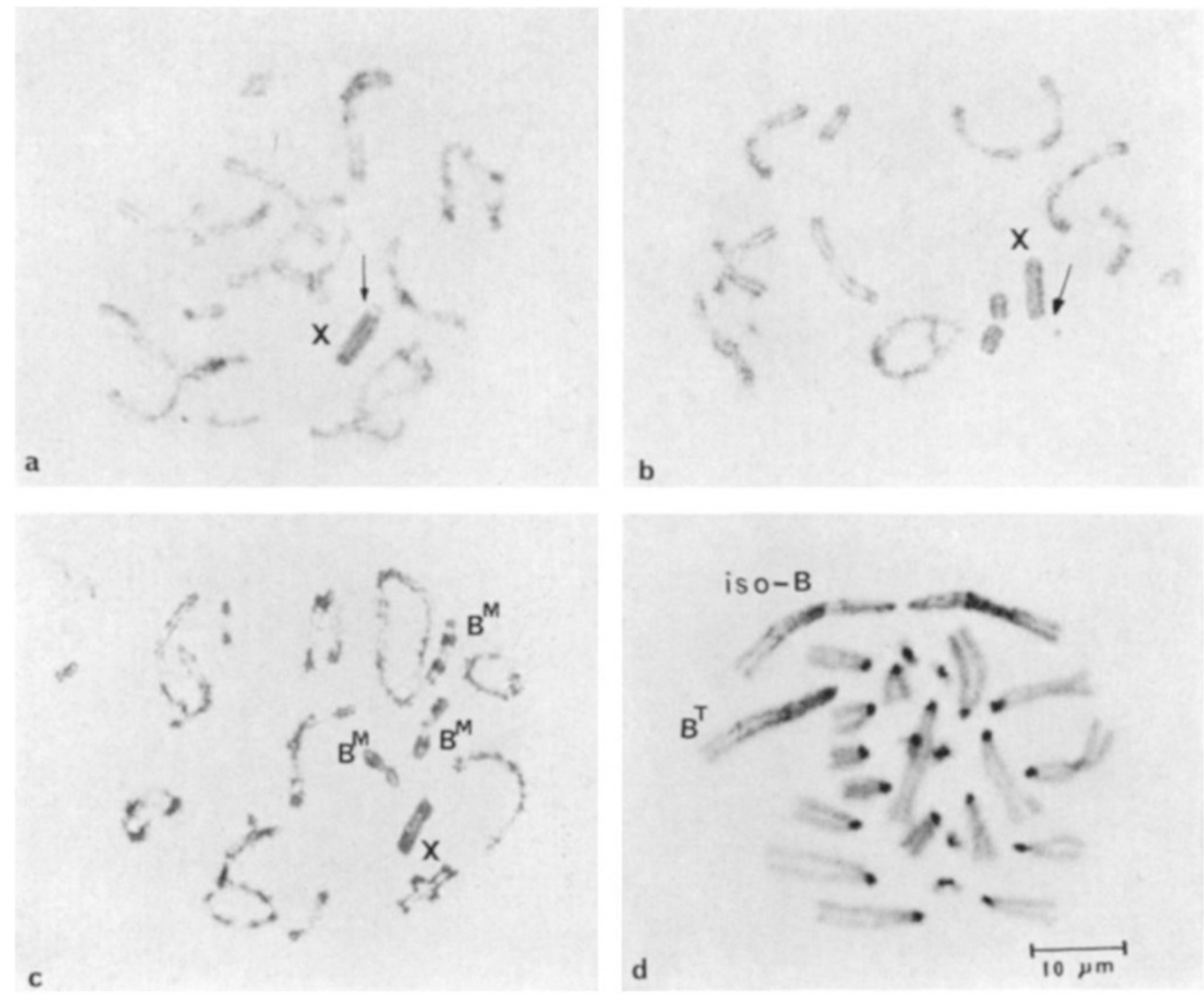

Plate II

FIr. 2.-X and B-chromosomes in the genus Phaulacridium.

(a) and (b). Orcein stained chromosomes of $P h$. vittatum (a) and $P h$. nanum (b) showing proximal $2^{\circ}$ constrictions.

(c) Differentially stained $\mathrm{B}^{\mathrm{M}}$-chromosome of $\mathrm{Ph}$. marginale.

(d) C-banded metacentric isochromosome in $P h$. vittatum male embryo which also has a $\mathrm{B}^{\mathrm{T}}$-chromosome. 
contrast to the similarities observed in two other Orthopteran speciesMyrmeleotettix maculatus (Gallagher et al., loc. cit) and Tettigidea lateralis (Fontana and Vickery, 1975). In both of these species the G-banding pattern of the X and B's are identical or sufficiently similar to support hypotheses of the presumed evolutionary origin of the B from a sex chromosome. The different banding patterns of B- and X-chromosomes observed in $P$. vittatum, like those in another Australian grasshopper Ch. terminifera (Webb, 1976) could be interpreted to support the suggestion that the origin of the B-chromosome from the X, or an A-chromosome, occurred sufficiently far back in the evolutionary history of the species (genus) to allow for subsequent divergence of the DNA contained in them.

Although the large B-isochromosome we have reported above is the first known instance of this particular type of B-chromosome in the genus Phaulacridium and adds to the B-chromosome types already known (see Westerman, 1977), the process of centric misdivision by which it was probably generated is perhaps fairly common. Thus the small B-isochromosome of $P h$. marginale was probably similarly derived from a small telocentric B.-chromosome. Nor would centric misdivision appear to be restricted to the B-chromosomes. It would appear from the present study that a similar process has occurred in the smallest chromosome in the complement-the $\mathrm{S}_{11}$. The $\mathrm{C}$-banding pattern of the metacentric $\mathrm{S}_{11}$ chromosome shown in fig. 1d is similar on both sides of the centromere. This would suggest that the $\mathrm{S}_{11}$ chromosome previously reported from La Trobe and other populations as carrying a supernumerary heterochromatic segment in meiotic cells may well be in error. It is more likely that the $S_{11}$ is an isochromosome, the heterochromatic nature of one arm at meiotic prophase being due to heterochromatinisation of the "extra" $\mathrm{S}_{11}$ chromosome present in the cell over and above the disomic number. Such heterochromatinisation of "extra " autosomes is known for both $\mathrm{S}_{11}$ trisomics of the related species $P h$. marginale (Westerman, unpublished data) and for polysomics in other grasshoppers (see Hewitt and John, 1968). Such an interpretation would explain the apparent lack of effects of $\mathrm{S}_{11}$ "segments" on chiasma frequencies in Ph. vittatum and Ph. marginale.

Acknowledgments.-We would like to thank $\mathrm{Mr}$ Peter Neuhaus for his valuable technical assistance and Ms Meg Powell for drawing fig. 3.

\section{REFERENCES}

BAVERSTOCK, P. R., WATTS, H. S., AND HOGARTH, J. T. 1976. Heterochromatin variation in the Australian rodent Uromys caudimaculatus. Chromosoma (Berl.), 57, 297-403.

BAVERSTOCK, P. R., WATtS, H. S., HOGARTH, J. T., ROBINSON, A. C., AND ROBINSON, J. F. 1977. Chromosome evolution in Australian rodents. II. The rattus group. Chromosoma (Berl.), 61, 227-241.

FontANA, P. G., AND viCKery, v. R. 1975. The B-chromosome system of Tettigidea lateralis (Say). II. New karyomorphs, patterns of pycnosity and giemsa banding. Chromosoma (Berl.), 50, 371-391.

Gallagher, A., HeWITt, G. M., AND GiBSON, I. 1973. Differential giemsa staining of heterochromatic B-chromosomes in Myrmeleotettix maculatus (Thunb.) (Orthoptera : Acrididae). Chromosoma (Berl.), 40, 167-172.

HewrTt, G. M., AND John, B. 1968. Parallel polymorphism for supernumerary segments in Chorthippus parallelus (Zetterstedt). I. British populations. Chromosoma (Berl.), 25, 319-342. 
JACKson, w. D., AND Cheung, D. s. M. 1967. Distortional meiotic segregation of a supernumerary chromosome producing differentia frequencies in the sexes in the shorthorned grasshopper Phaulacridium vittatum. Chromosoma (Berl.), 23, 24-37.

ROWE, H., AND WESTERman, M. 1974. Population cytology of the genus Phaulacridium $I$. Phaulacridium vittatum (Sjöst)-Australian mainland populations. Chromosoma (Berl.), $46,197-205$.

SHAW, D. D., WEBB, G. C., AND WILKINson, P. 1976. Population cytogenetics of the genus Caledia (Orthoptera : Acridinae). II. Variation in the pattern of C-banding. Chromosoma (Berl.), 56, 169-190.

WEBB, G. C. 1976. Chromosome organization in the Australian plague locust, Chortoicetes terminifera. I. Banding relationships of the normal and supernumerary chromosomes. Chromosoma (Berl.), 55, 229-249.

WEBB, G. C., AND KOMAROWSKI, L. 1976. Haplo-diploid locust embryos arising by accidental thelytoky in Chortoicetes terminifera investigated by G-banding. Chromosoma (Berl.), $55,247-251$.

westerman, M. 1975a. Population cytology of the genus Phaulacridium. III. Phaulacridium marginale (Walker); Polymorphism for extra heterochromatin. Heredity, 34, 11-28.

westerman, M. 1975b. Population cytology of the genus Phaulacridium. IV. Ph. marginaleThe North Island populations. Heredity, 35, 165-172.

westerman, m. 1977. Population cytology of the genus Phaulacridium. V. Ph. marginaleThe Omarama population. Aust. 7. Biol. Sci., 30, 319-328.

Westerman, M., AND Dempsey, J. 1977. Population cytology of the genus Phaulacridium. VI. Seasonal changes in the frequency of the B-chromosome in a population of $P h$. vittatum. Aust. 7. Biol. Sci., 30, 329-336. 